\title{
ML Decoding of Block Codes on their Tailbiting Trellises
}

\author{
Priti Shankar, P.N.A. Kumar, K.Sasidharan \\ Department of Computer Science and Automation, \\ Indian Institute of Science, Bangalore 560012. \\ email: \{priti,pnakumar\}@csa.iisc.ernet.in
}

\begin{abstract}
A maximum likelihood decoding algorithm is presented for tailbiting trellises for block codes. The algorithm works in two phases. The first phase is a Viterbi decoding algorithm on the tailbiting trellis, while the second uses the $\mathbf{A}^{*}$ algorithm adapted for application in this context. Results of simulations on tailbiting trellises for some block codes, indicate that this decoding algorithm is quite fast.
\end{abstract}

\section{INTRODUCTION}

Tailbiting trellises for convolutional codes were originally introduced by Solomon and van Tilborg[4] and for block codes in[1]. Approximate decoding algorithms on tailbiting trellises were proposed by several authors, and most recently by Shao et al.[3]. In this paper we describe a maximum likelihood two phase decoding algorithm proposed by the authors in [2] and study its performance on a few codes. Section II presents the decoding algorithm and section III presents the results of simulations.

\section{Decoding on Tailbiting Trellises}

The structure of a tailbiting trellis can be described as follows. Let there be $l$ start (equivalently final) states. Each start state corresponds to a subcode, which consists of all codewords that begin and end at that state. Thus there are a total of $l$ subcodes. These subcodes all have identical subtrellises which differ only in edge labels. The subtrellis containing the all 0 codeword defines a subcode which is a group, and the remaining subtrellises correspond to cosets of the group. The $l$ subtrellises share states at various time indices. The structure exhibited by tailbiting trellises can be exploited to give a decoding algorithm that is now described. One possibility that exists for exact decoding on a tailbiting trellis, is to perform Viterbi decoding on each of the $l$ subtrellises and then choose the codeword with minimal cost among the $l$ survivors at time index $n$ in each subtrellis. (From now on, we call these winning codewords). However, we aim at an exact algorithm that is more efficient. Let us term the codeword that would be the final survivor if Viterbi decoding was performed on subtrellis $T_{j}$ alone, as the $T_{j}$-codeword survivor. If one performed a Viterbi decoding algorithm on the tailbiting trellis, then survivors at the $l$ final states need not be codewords. Let the set of start states be $s_{1} \ldots s_{l}$ and the set of final states $f_{1} \ldots f_{l}$. We term a survivor on the tailbiting trellis, which corresponds to an $s_{i}-f_{i}$ path, a codeword survivor, and one that corresponds to a $s_{i}-f_{j}$ path, $i \neq j$, a non-codeword survivor. The algorithm, which is a two phase algorithm, is described below. Phase 1.

Execute a Viterbi decoding algorithm on the tailbiting trellis, and obtain survivors at each node. If the path with minimum

${ }^{1}$ This work was partly supported by DST, India, through the grant No.III.5(31)/99-ET to B.S.Rajan.

\author{
B.S. Rajan ${ }^{1}$ Department of Electrical \\ Communication Engineering, Indian Institute of \\ Science, Bangalore 560012. \\ email:bsrajan@ece.iisc.ernet.in
}

cost corresponds to a codeword survivor, decoding is complete. If no such codeword survivor exists, go to Phase 2.

\section{Phase 2}

1. Consider only subtrellises $T_{j}$ such that the winning path at $T_{j}$ is a non-codeword path, and such that there is no codeword winner with smaller cost. These are the residual trellises. Initialize a sequence $P_{j}$ for each residual trellis $T_{j}$ to the empty sequence. The sequence $P_{j}$, in fact stores the current candidate for the shortest path in trellis $T_{j}$. Let the estimate $h\left(s_{j}, f_{j}\right)$ associated with the empty path be the cost of the survivor at $f_{j}$ obtained in the first phase.

2. Let $j$ be the index of the subtrellis with the minimum value of the estimate, i.e. with the non-codeword survivor of minimum cost. Run the $A^{*}$ algorithm on trellis $T_{j}$, (called the current trellis). For a node $u$, take $h\left(u, f_{j}\right)$ to be $h\left(s_{i}, f_{j}\right)-$ $\operatorname{cost}($ survivor $(u))$ where cost (survivor $(u))$ is the cost of the survivor at $u$ obtained in the first phase.

3. At each step, compare $p(u)+h\left(u, f_{j}\right)$ (called the potential of node $u$ ) in the current subtrellis and the potential of the current best node in all other subtrellises. If at any step the former exceeds the latter, associated with subtrellis, say, $T_{k}$, then make $T_{k}$ the current subtrellis, storing the state of the suspended computation on $T_{j}$, and run the $A^{*}$ algorithm on $T_{k}$ either from start node $s_{k}$ (if $T_{k}$ was not visited earlier) or from the node which it last examined in $T_{k}$. Stop when the goal vertex is reached in the current subtrellis.

\section{Simulation}

Simulations were carried out on the tailbiting trellises of several linear block codes. For the tailbiting trellis of the Golay $(24,12)$ code, the exact algorithm on the tailbiting trellis ran about 6 times as fast as the Viterbi algorithm on the conventional trellis on an AWGN channel at a SNR of $2.5 \mathrm{db}$. Our simulation results indicate that the second pass consumes negligible time at SNR's of 2.5 and beyond. The space required includes that used to store the results of the first pass and also arrays to include the information about whether a node in the tailbiting trellis is a member of a particular subtrellis or not.

\section{REFERENCES}

[1] A.R.Calderbank, G.David Forney,Jr., and Alexander Vardy, Minimal Tail-Biting Trellises: The Golay Code and More, IEEE Trans. Inform. Theory 45(5) July 1999,pp 1435-1455.

[2] K.Deshmukh, P. Shankar, A. Dasgupta and B. Sundar Rajan, On the many faces of block codes, in Proceedings of STACS 2000, LNCS 1770, (Lille, France, February 2000), pp 53-64.

[3] R.Y.Shao, Shu Lin and M.P.C. Fossorier, Decoding of Codes Based on Their Tail Biting Trellises, in Proc. 2000 Int. Symp. Inform. Theory, Sorrento,Italy, pp 342.

[4] G.Solomon and H.C.A. van Tilborg, A connection between block and convolutional codes, SIAM J. Appl. Math.,37, October 1979 , pp 358-369. 